\title{
Monoglycerides oleogels as fat replacers in filling creams for sandwich cookies
}

\author{
Camila A. Palla ${ }^{1,2}$, María F. Wasinger ${ }^{1}$, and María E. Carrín ${ }^{1,2}$ \\ ${ }^{1}$ Departamento de Ingeniería Química, Universidad Nacional del Sur (UNS) \\ ${ }^{2}$ Planta Piloto de Ingeniería Química - PLAPIQUI (UNS-CONICET) \\ Bahía Blanca, Argentina \\ cpalla@plapiqui.edu.ar
}

\begin{abstract}
BACKGROUND

Many food products need to be reformulated to reduce the intake of saturated and trans fats which are considered unhealthy. In particular, the reformulation of filling creams (FC) is challenging as these fats cannot be directly replaced with liquid oil without affecting the final product properties. This research studied the formulation and characterization of FC for sandwich cookies using monoglycerides oleogels as fat material.
\end{abstract}

\section{RESULTS}

FC formulated with $260 \mathrm{~g} \mathrm{~kg}^{-1}$ oleogel showed viscoelastic moduli values that did not differ significantly from those measured in a commercial filling cream used as reference (FC-CSC). The oil binding capacity of FC decreased with the increase of oleogel content. The increase of the oleogel amount in the formulation produced a decrease in hardness but an increase in adhesiveness and cohesiveness. Hardness, adhesiveness, and cohesiveness ranged from 0.66 to $3.48 \mathrm{~N}, 0.44$ to $0.86 \mathrm{~N} . \mathrm{s}$, and 0.07 to 0.29 , been through the copyediting, typesetting, pagination and proofreading process which may lead to differences between this version and the Version of Record. Please cite this article as doi: $10.1002 /$ jsfa.10863 
respectively. When FC were used for assembling cookies into sandwiches, an oil loss of about $9 \mathrm{~g} \mathrm{~kg}^{-1}$ FC after 21 days of storage was found in FC containing 220 and $260 \mathrm{~g} \mathrm{~kg}^{-}$

${ }^{1}$ oleogel. The nutritional improvement due to the use of oleogels in FC led to a reduction in saturated fatty acids between $64.5 \%$ and $35.2 \%$ and from 1.0 to $0.0 \%$ trans fatty acids in comparison with FC-CSC.

\section{CONCLUSION}

Full fat replacement with monoglycerides oleogels in FC formulations allows the obtention of products with good quality and some similar characteristics to those obtained for FC-CSC, with the added benefit of a healthier nutritional profile.

Keywords: Fat replacer; Oleogels; Textural properties; Filling cream; Cookies. 
Corresponding author: TE: (54-291) 4861700 Int 239, Fax: (54-291) 4861600. E-mail address: cpalla@plapiqui.edu.ar

\section{Introduction}

Cookies are part of the daily diet of many people worldwide. Convenience in consumption and portability has made cookies a popular "on-the-go" snack among consumers, mainly between children and adolescents. ${ }^{1,2}$ In particular, sandwich cookies consisting of two cookies with a filling in-between are widely consumed due to their texture and flavor attributes. ${ }^{3}$ According to a recent report, the sandwich cookies market is growing at the fastest pace within the Global Sweet Biscuit Market, which is expected to grow at a Compound Annual Growth Rate (CAGR) of 5.35\% during the forecasted period (2020-2025). ${ }^{3}$ Creams for various fillings are often made with a combination of highly saturated hard stock fat -which could include trans fatty acids- and unsaturated oils, along with other ingredients such as sugar and flavorings. ${ }^{4,5}$ Since the consumption of excessive amounts of trans and saturated fats is considered unhealthy, there is a growing need to replace or reduce the use of these fats in food formulations by developing products with similar physical and organoleptic characteristics but healthier for consumers. ${ }^{6}$ In fact, the elimination of industrially-produced trans fats from the global food supply has been identified as one of the priority targets of the World Health 
Organization's strategic plan 2019 - 2023. Under the denomination REPLACE, an action package has been provided that promotes, among others, the replacement of industrially-produced trans fat with healthier fats and oils. ${ }^{7}$

Oil structuring or oleogelation strategies aimed at creating soft matter structures with the functionality of fats, but with the nutritional profile of liquid oils. ${ }^{8}$ Among the wide number of different molecules which are able to structure oils, monoglycerides (MG) and waxes are some of the most promising alternatives for replacing hard stock structured fats. ${ }^{9}$ The formation of a three-dimensional gel network which mimics triglycerides crystallization is accomplished through molecular self-assembly of crystalline particles. ${ }^{10}$ Although oleogels’ physicochemical characterization remains the subject of many studies, their application in different food products has become the focus of most recent researches. The potential use of oleogels as fat replacers has been studied in ice creams, chocolate spreads, sweet breads, cakes, cookies, muffins, maize tortillas, pâté, beef burgers, and frankfurters sausages. ${ }^{9,11-16}$ Particularly, Stortz et al. ${ }^{4}$ evaluated the use of ethylcellulose oleogels combined with interesterified hydrogenated palm oil to prepare filling creams. The authors found that by gelling the liquid oil portion of the cream it was possible to reduce the oil migration compared to the traditional creams made with liquid oil. Thus, this work showed that oleogelation can be an effective way to prolong the shelf-life of sandwich cookies since oil leaks can lead to softening of the cookies, which is considered a texture defect. However, another outstanding aspect is that organogelation can be used for the replacement of saturated and trans fats offering a greatly improved fatty acid profile for the creams. More 
recently, Tanti et al. ${ }^{17}$ investigated the partial and full substitution of icing shortening with freeze-dried hydroxypropyl methylcellulose (HPMC) and methylcellulose (MC) structured canola oil in sandwich cookie creams as a strategy to replace fats rich in saturated and trans fatty acids. They found that the full replacement resulted in creams that were excessively hard, suggesting the partial substitution to reach desirable functionality. However, physical properties of oleogels as well as their application in foods depend on the molecules used for structuring oil. As a consequence, more studies are required about the formulation of filling creams for sandwich cookies using other types of oleogels that consider the effects of full fat substitution on product quality. Keeping the aforementioned perspective in mind, in the present research the use of oleogels of monoglycerides, mainly monostearin, and high oleic sunflower oil as fat replacers in filling creams for sandwich cookies was studied. To this purpose, an analysis of formulation and preparation conditions of filling creams was performed in order to obtain a material with similar properties to a filling cream of commercial sandwich cookies, which was used as reference material. Filling creams containing different proportions of monoglycerides oleogels were characterized by using different techniques, including rheology, textural properties, oil binding capacity (OBC), and color. Furthermore, the creams were used for assembling commercial cookies into sandwiches with the aim to evaluate the quality of the obtained products by texture analysis and oil migration on time. Innovative aspects of this contribution include the full replacement of hard fats for oleogels of monoglycerides in the elaboration of filling 
creams as well as the application of a novel technique that allows to determine the texture properties of sandwich type cookies.

\section{Materials and methods}

\subsection{Materials}

Myverol 18-08 NP was generously donated by Kerry (Ireland), its product specification being: monoglycerides $>90 \%$ purity grade and $72.0^{\circ} \mathrm{C}$ melting point. Refined high oleic sunflower oil (HOSO) was bought at a local supermarket. The main fatty acids (FA) weight composition of the Myverol obtained by GLC (see 2.2 section) was: stearic acid $906.5 \pm 1.2 \mathrm{~g}$ kg- 1 , palmitic acid $64.3 \pm 0.2 \mathrm{~g}$ kg- 1 , and arachidic acid $15.3 \pm 0.3 \mathrm{~g} \mathrm{~kg}-1$ The FA composition of HOSO classified according to the degree of saturation was: saturated FA $72.5 \pm 1.2 \mathrm{~g}$ kg-1, monounsaturated FA $877.8 \pm 1.0 \mathrm{~g} \mathrm{~kg}-1$, and polyunsaturated FA $49.7 \pm 0.4$ g kg-1. Granulated sugar (sucrose 99\%, cornstarch $0.5 \%$, and sodium alginate $0.02 \%$ ) was obtained from Taxonera (Argentina). Commercial sandwich cookies (CSC) (Rumba, Bagley, Argentina) were used to obtain an appropriate filling cream for use as reference (FC-CSC), which was produced using beef fat as fat material. Commercial cookies without filling cream (Chocolinas, Bagley, Argentina) were selected due to their similar size and shape to CSC. Both types of cookies, fat-free powdered milk, and vanilla essence were purchased from local stores. Fatty acid methyl esters (FAME) standards were purchased from Supelco (Bellefonte, USA). All the other reagents, gases, and solvents used in gas-liquid chromatography (GLC) were of analytical or chromatographic grade. 


\subsection{Determination of FA composition}

The composition of FA in the acylglycerols of HOSO, Myverol, and fat extracted from the filling cream of CSC was determined by GLC. Samples were subjected to cold transesterification treatment to obtain FAME which were analyzed in a 7820A series gas chromatograph (Agilent) according to AOCS Official Methods Ce2-66 and Ce1-62 18.

The extraction of fat from FC-CSC was carried out according to ISO 17189- IDF 194 Reference Method ${ }^{19}$ using petroleum ether as solvent.

\subsection{Oleogel preparation}

Oleogels were prepared according to the methodology described by Palla et al. ${ }^{20} \mathrm{We}$ replicated this procedure since it allows to produce oleogels with similar properties to a commercial margarine. ${ }^{11,20}$ In brief, $100 \mathrm{~g} \mathrm{~kg}^{-1}$ of Myverol was solubilized in HOSO previously heated at $80{ }^{\circ} \mathrm{C}$ using a glass container system with a temperature-controlled water bath. The mixture was kept under magnetic agitation for $30 \mathrm{~min}$ and subsequently put into rectangular polyethylene containers at $5{ }^{\circ} \mathrm{C}$ to allow gel formation under static conditions. Oleogels were stored in darkness at $5{ }^{\circ} \mathrm{C}$ for $48 \mathrm{~h}$ before using to prepare creams.

\subsection{Filling creams preparation}

Filling creams were formulated using variable amounts of oleogel according to proportions presented in Table 1. Granulated sugar was milled to obtain powder sugar and after sieving, the fraction corresponding to particle size $<149 \mu \mathrm{m}$ was selected in order to obtain creams with a visually homogeneous appearance. To prepare the filling 
creams, powder sugar and fat-free milk were mixed in a blender. After that, oleogel and vanilla essence were added and manually homogenized for $5 \mathrm{~min}$ at room temperature, and later stored at $18{ }^{\circ} \mathrm{C}$ in hermetic containers. The obtained filling creams were identified according to oleogel content as FC-22 (220 g kg-1), FC-26 (260 g kg $\left.{ }^{-1}\right)$, FC-30 (300 $\mathrm{g} \mathrm{kg}^{-1}$ ), and FC-40 (400 $\mathrm{g} \mathrm{kg}^{-1}$ ) (Fig. 1). The physical properties of FC were measured after $72 \mathrm{~h}$ of storage unless otherwise specified.

Prior to selection of the mentioned procedure, the mixture and homogenization of the components at a temperature around $50{ }^{\circ} \mathrm{C}$ was assayed. However, the resulting creams showed a heterogeneous appearance and undesirable color. As a consequence, this preparation procedure was discarded.

\subsection{Measurement of filling cream properties}

\subsubsection{Rheological properties}

Rheological properties of filling creams were investigated by dynamic oscillatory tests using a Paar Physica rheometer (model MCR 301, Anton Paar GmbH, Austria) equipped with a parallel-plate geometry (50 mm diameter). A thin layer of sample was spread over the lower plate and the measuring gap was set to $1 \mathrm{~mm}$. The following tests were performed at $20^{\circ} \mathrm{C}$ : i) a frequency sweep test at fixed strain $(0.01 \%)$ where the frequency was increased from 5 to $100 \mathrm{rad} \mathrm{s}^{-1}$, and ii) a strain sweep test at a fixed frequency $\left(10 \mathrm{rad} \mathrm{s}^{-1}\right.$ ) with a strain range from 0.01 to $100 \%$. Frequency sweep measurements were performed in the linear viscoelastic region checked in a stress sweep; after that, the strain was set at $0.01 \%$. The tests were performed $1 \mathrm{~h}$ after the preparation of creams and replicated at least three times for each sample. 


\subsubsection{Texture analysis of filling creams}

The textural properties of the filling creams were determined by texture profile analysis (TPA) of a two-cycle compression test using a Texture Analyzer TA Plus (Lloyd Instruments, England) equipped with a $50 \mathrm{~N}$ load cell. Each filling cream was introduced into at least five cylinders (15 mm diameter and $15 \mathrm{~mm}$ high), stored at 18 ${ }^{\circ} \mathrm{C}$ for $72 \mathrm{~h}$, and carefully removed from containers to obtain cylindrical samples. The samples were compressed to $50 \%$ of their original height using a cylindrical probe (50 mm diameter) at a crosshead speed of $1 \mathrm{~mm} \mathrm{~s}^{-1}$. From the resulting force-time curve, three mechanical parameters were determined: i) hardness (HA), the height of the force peak on the first compression cycle, ii) cohesiveness (CO), the ratio of the positive force areas under the second and first compressions, and iii) adhesiveness (AD), the negative force area of the first cycle, which represents the work necessary to pull the compressing plunger away from the sample. ${ }^{21}$

\subsubsection{Oil binding capacity (OBC) of filling creams}

The ability of formulated and commercial creams to retain oil contained in their matrix was tested using a simple centrifugation method which is often used for oleogels evaluation. Each filling cream was introduced into five eppendorf tubes (1 g of sample per tube) and centrifuged using a microcentrifuge (Giumelli z-127-D, Argentina) at $9650 \times \mathrm{g}$ for $15 \mathrm{~min}$ and $5{ }^{\circ} \mathrm{C}$ to avoid heating. OBC was calculated as a function of the percentage of oil released from the sample after centrifugation:

$$
O B C(\%)=100-\left(\frac{\text { mass of realeased oil }}{\text { mass of filling cream }}\right) 100
$$

\subsubsection{Color}


The measurement of color of the filling creams was performed in a HunterLab UltraScan XE tristimulus colorimeter (Hunter Associates Laboratory, Inc., Reston, VA). The measurements were done by duplicate in three independent samples. The total color reflected by the sample was expressed in accordance with the CIELAB system at $10^{\circ}$ observer angle with D65 illuminant. The parameters measured were $L^{*}$ [lightness: $0=$ black, $100=$ white], $a^{*}$ [greenness $(-)$, redness $(+)$ ] and $b^{*}$ [blueness $(-)$, yellowness $(+)$ ]. The total color difference $\left(\Delta E^{*}\right)$ between each FC and the FC-CSC was calculated taking into account the differences between their average color parameters as follows: $\Delta E^{*}=\left(\left(\Delta L^{*}\right)^{2}+\left(\Delta a^{*}\right)^{2}+\left(\Delta b^{*}\right)^{2}\right)^{1 / 2}$. A value of $\Delta E^{*}<3$ means that the differences are not visible to the human eye. ${ }^{22}$

In addition, the Whiteness index (WI), which represents the overall whiteness of a food product, was calculated according to the following formula: $W I^{*}=100-$ $\left(\left(100-L^{*}\right)^{2}+a^{* 2}+b^{* 2}\right)^{1 / 2} \cdot 23$

\subsection{Measurement of sandwich cookies properties}

\subsubsection{Assembly of cookies}

The ability of prepared FC and FC-CSC to assemble cookies was evaluated by spreading $0.06 \mathrm{~g} \mathrm{~cm}^{-2}$ of the filling cream on one cookie and topping off with another to form a sandwich. That ratio of FC mass/cookie area was selected based on the values obtained from CSC. Samples were stored in hermetic containers at $18{ }^{\circ} \mathrm{C}$ for $72 \mathrm{~h}$ until analysis, unless otherwise specified. Manual inspection was used to check the adhesion of the parts.

2.6.2 Texture analysis of sandwich cookies 
To determine the tangential force required to separate two cookies attached by filling cream, a specific fixture for a Texture Analyzer TA Plus (Lloyd Instruments, England) was designed and built using a fused filament fabrication (FFF) 3D printer (method under publication). During the assay, one of the cookies composing the sandwich cookie was pushed at a crosshead speed of $1 \mathrm{~mm} / \mathrm{s}$ until achieving its complete separation. From the resulting force-time curve, two mechanical parameters were determined: the maximum force value (Fmax) and the area under the curve (W), which represents the work necessary to separate the cookies. At least 8 replicates were tested for each type of assembled cookie. Furthermore, the commercial sandwich cookie was analyzed for comparison purposes.

\subsubsection{Oil loss (OL) determination}

To test the oil migration from filling creams, sandwich cookies were placed on round filter papers (Whatman \#4) into petri dishes. The amount of oil lost by each sample was determined by the difference in weight of the filter paper before (initial paper wt) and after placing the sandwich cookie on it (paper wt) for $4,7,15$, and 22 days at $18{ }^{\circ} \mathrm{C}$. A filter paper with two cookies (without filling cream) on it was used as control in the experiments to account for the cookie fat effect on the filter paper. At least four replicates were prepared. OL was calculated for each time as:

$$
O L(\%)=
$$

$\left(\frac{(\text { paper wt }- \text { initial paper wt })-(\text { control paper wt }- \text { initial control paper wt })}{\text { mass of filling cream }}\right) 100$

\subsection{Statistical Analysis}


Results are reported as mean \pm standard deviation of the number of replicate measurements in one representative experiment of at least three independent experiments. Statistical analysis was carried out by one-way ANOVA using OriginPro 9.1 software (OriginLab, USA). Fisher's post test was used for multiple comparisons between means with a significance level $\mathrm{p}$-value $\leq 0.05$.

\section{Results and Discussion}

\subsection{Physicochemical properties of filling creams}

\subsubsection{Fatty acid composition}

The fat content for FC-CSC obtained by solvent extraction was $223.6 \pm 13 \mathrm{~g} \mathrm{~kg}^{-1} \mathrm{FC}$, being their composition: saturated FA $497.1 \pm 6.0 \mathrm{~g} \mathrm{~kg}^{-1}$, monounsaturated FA $453.2 \pm$ $3.9 \mathrm{~g} \mathrm{~kg}^{-1}$, and polyunsaturated FA $49.7 \pm 1.2 \mathrm{~g} \mathrm{~kg}^{-1}$. Palmitic acid was found to be the main saturated FA component (230.4 $\left.\pm 3.0 \mathrm{~g} \mathrm{~kg}^{-1}\right)$ followed by stearic acid (203.6 \pm 2.3 $\mathrm{g} \mathrm{kg}^{-1}$ ), which was in agreement with that previously reported for beef tallow fat. ${ }^{24}$ Trans FA was also found in the filling cream composition of CSC in an amount of 46.7 $\pm 1.2 \mathrm{~g} \mathrm{~kg}^{-1}$.

As was expected, the filling creams prepared with oleogels showed a significantly reduced content of saturated FA and an increased content of monounsaturated FA (Table 1). The improvement in the nutritional profile due to the use of oleogels in filling cream formulation was a reduction of saturated FA of $64.5 \%, 58.0 \%, 52.3 \%$, and $35.2 \%$ for FC-22, FC-26, FC-30, and FC-40, respectively, and 0.0\% trans FA (traces are 
present in the vegetable HOSO composition). Another highlighted aspect was the fact that the reformulation allowed to replace fats of animal origin with vegetable fats.

\subsubsection{Rheological properties of filling creams}

Small deformation rheology was used to characterize FC-CSC and FC formulated with different amounts of oleogel. The elastic modulus ( $\left.\mathrm{G}^{\prime}\right)$ behavior resulted independent of the frequency for all the samples (Fig. 2A) and the ratio G"/G' $(\tan \delta$ ) resulted lower than 1 throughout the entire range of applied frequency (Table 2), indicating a predominant 'solid-like' behavior of the creams. ${ }^{25}$ At an intermediate frequency (20 $\mathrm{rad} / \mathrm{s}$ ), G' value of FC-CSC was $2.06 \times 10^{6} \pm 3.55 \times 10^{5} \mathrm{~Pa}$ and no significant differences ( $p>0.05$ ) were found between FC-CSC and FC-26, which showed a value of $1.78 \times 10^{6} \pm$ $1.53 \times 10^{5} \mathrm{~Pa}$. Based on statistical analysis, G' increased with the decrease of oleogel content in the formulations $(\mathrm{p}<0.05)$, indicating a more solid-like behavior of creams, that is, a stronger food matrix. Since the decrease in oleogel content was accompanied by an increase in sugar content, this behavior could be attributed to the higher interparticulate interactions among sugar dispersed particles. Babin et al. ${ }^{26}$ investigated the interactions of sugar particles dispersed in different fat systems, in the presence and absence of emulsifiers. The increment of sugar concentration in these systems caused the viscosity to increase too. The sugar crystals revealed a stronger interaction with the tested vegetable oils while the presence of emulsifiers (monoolein) made the sugar particles to disperse better in the oil, resulting in a viscosity decrease. It can be speculated that monoglycerides may also have modified the formation of hydrophilic adhesive contacts between sugar particles. As a consequence, both increment of sugar 
content and the reduction of monoglycerides content could have affected the structuration level, increasing G' value.

The comparison between FC-CSC and FC-22, both with the same fat content, showed that FC-22 presented a higher G' value and a higher tan $\delta$ average value, indicating not only a higher mechanical strength, but also a more elastic-dominant behavior. In fact, FC-22 showed a $\tan \delta$ value lower than 0.1 , which means that this cream satisfied the rheological definition of a strong gel. ${ }^{25}$ The remaining samples showed that $\tan \delta$ increased with oleogel content, indicating a greater contribution of the viscous component for these creams. On the other hand, Fayaz et al. ${ }^{9}$ reported a $G^{\prime}$ value of 4.9 $\times 10^{6} \mathrm{~Pa}$ for a chocolate spread formulated with $40 \%$ of fat composed by a mixture of MG oleogel and palm oil, which is similar to that obtained for FC containing the lowest amount of oleogel. This comparison could indicate that the full replacement of hard fats for MG oleogels is possible when it is accompanied by changes in component compositions.

The dynamic strain test was performed to evaluate the mechanical stability of structured filling cream formulations and the reference cream. The curves of FC showed similar patterns of a progressive decrease in the elastic modulus with the increase in the percentage of strain, with a more sharply structural breakdown in samples with less oleogel content (Fig. 2B). The test showed a slight difference in the linear viscoelastic regions between the different filling creams formulated with oleogel. An increase in the critical yield strain value -determined as the onset value of the $G^{\prime}$ curves- with the decrease in oleogel content was observed, being about $0.03 \%$ for FC-40 and $0.12 \%$ for 
FC-22, which indicated that a matrix with a more compact structure was obtained due to the increase in sugar content. FC-26 showed similar behavior to that obtained for FCCSC cream.

\subsubsection{Texture analysis of filling creams}

The values of hardness, adhesiveness, and cohesiveness of filling creams determined by the TPA test are shown in Table 2. Samples formulated with an incremental concentration of oleogel were significantly softer. In fact, the hardness and remaining textural parameters of FC-40 could not be determined using the compression test due to the softness of the material. The FC-22 samples were around 2.5 times less hard than the control cream, which indicated the high degree of structuration and stability that the saturated and trans fats provide to the food matrix and therefore, how difficult their replacement is. However, it is important to mention that hardness values between 0.41 and $1.49 \mathrm{~N}$ have been reported for commercial cookie creams ${ }^{17}$, which are more similar to those obtained for the FC produced in this work.

The adhesiveness of FC increased significantly as the oleogel content increased. For example, FC formulated with $300 \mathrm{~g} \mathrm{~kg}^{-1}$ of oleogel exhibited a 2-fold increase in AD with respect to the cream containing $220 \mathrm{~g} \mathrm{~kg}^{-1}$, indicating that in this case a greater proportion of sugar crystals was lubricated by the fat phase providing a better stick capacity. In comparison with the reference cream, all the produced FC showed significantly higher values of AD. This is a relevant result since the replacement of solid fats for a proportion of monoglycerides oleogel equal or higher, allowed to obtain creams with greater adhesiveness, which is an essential factor in the production of 
sandwich cookies. Tanti et al. ${ }^{17}$ reported lower values of $\mathrm{AD}, 0.43$ and 0.47 N.s, for filling creams produced with $40 \%$ of fat phase composed by HPMC and MC structured canola oil, respectively.

Regarding cohesiveness, the creams presented a behavior similar to that found in AD. The increase in oleogel content favored the arrangement of sugar particles within the food matrix in a more cohesive structure. The CO values of all FC were higher than that of the control cream and similar to those reported for other commercial cookie creams as well as creams formulated with a mixture of shortening and HPMC and MC oleogels. ${ }^{17}$

\subsubsection{Oil binding capacity (OBC) of filling creams}

The application of oleogels in filling creams requires that the oil present in the food matrix is not released. To test this, OBC was determined in FC by a centrifugationbased method. It can be seen from the data in Table 3 that FC showed significant differences in their OBC values $(\mathrm{p}<0.05)$. The highest values were obtained for FCCSC, FC-22 and FC-26 with average values between 100.06 and 99.85\%, in which free oil was practically not observed during the test. OBC decreased when oleogel content was increased in the filling cream formulation, $91.78 \%$ being the lowest value found in FC formulated with $400 \mathrm{~g} \mathrm{~kg}^{-1}$ of oleogel. It is interesting to note that the OBC value corresponding to the MG oleogel used in the formulations was $86.64 \pm 0.76 \%$, which indicated that an increase of this property was achieved by mixing of the oleogel with solid components. During oleogelation, the immobilization of liquid oil occurs due to the crystallization of monoglycerides, which lead to the formation of a three- 
dimensional gel network, depending on the interaction of crystals and crystalline aggregates. $^{27}$ The fact that oil retention has increased in FC suggested that sugar particles provided more surface area for the adhesion of oil weakly bound to the gel network. Thus, an interesting finding from OBC results is that some of the formulated FC had food matrices in which the oil was highly structured and as a consequence, they showed a behavior similar to the commercial cream.

\subsubsection{Color}

In order to evaluate the effect of oleogel content in the appearance of the creams, the color parameters were determined (Table 3). The predominant white color of creams was detected by high $L^{*}$ values. No significant differences in $L^{*}$ between FC-CSC and FC prepared with oleogel were found with the exception of FC-40, which was different from all other samples. The highest oleogel content resulted in an increase in the cream luminosity. Contrarily, the absolute values of $a^{*}$ and $b^{*}$ parameters of filling cream containing oleogels were significantly lower than the reference values, indicating that formulated creams showed a less green tone and a lower yellowish color. Furthermore, in general, no significant differences were found in these parameters among the different formulated FC. According to the $\Delta E^{*}$ values, the total color differences between the reference cream and each of the formulated FC would be appreciated by the human eye. On the other hand, the FC exhibited whiteness indices higher than that obtained for the commercial cream, which is an interesting finding, taking into account the consumers' preferences for creams with white color. These color differences can be explained taking into account that FC-CSC and oleogel-based creams were produced 
with different ingredients and compositions. The fact that creams containing oleogel showed a less yellow and whiter tone could be associated with the HOSO that has little coloration, as well as to the sugar used. Although, remaining minor components such as milk or vanilla essence could also contribute to the color appearance.

\subsection{Quality analysis of assembled sandwich cookies}

The formulated FC and the reference cream were used for assembling cookies. After 72 hours of storage, it was manually checked that the two parts remained attached by the cream's action. As a result of this test, it was observed that all the assembled cookies were strongly attached with the exception of those prepared using FC-22. This was an unexpected result since, based on AD values, FC-22 showed a greater adhesion to the probe than the control cream. Probably, the oleogel content of FC-22 was not enough to generate a cohesive matrix with adequate adhesion to cookie surfaces. It was consistent with the more granular appearance presented for FC-22 (Fig. 1). As a consequence, that system was not taken into account for carrying out the quality analysis of assembled sandwich cookies.

\subsubsection{Texture analysis of sandwich cookies}

Fig. 3 shows a schematic representation of the procedure used to determine the textural properties of assembled cookies (Fig. 3A) and representative curves obtained from this test (Fig. 3B). The characteristic parameters determined from the curves, Fmax and W, are shown in Table 4. It was found that significantly higher values of tangential forces were required to take off the cookie in those sandwich cookies assembled with FC formulated with lower MG oleogel content. The highest Fmax value was obtained for 
FC-CSC; however, all sample values were lower than that of CSC. Regarding the work required to separate the cookie, it increased with the increase in the oleogel content in FC and non-significant differences were found between FC-40 and CSC and between FC-26, FC-30, and FC-CSC. These results agree with the previous findings of adhesiveness, showing that the higher the adhesiveness of FC is, the better the attachment between cookies. On the other hand, as can be observed from the curves, FC-CSC and CSC samples showed a fracturability point, represented by a breakpoint in the curve. Usually, fracturability is displayed by a product of a high degree of hardness and a low degree of cohesiveness ${ }^{21}$, which is in agreement with the results of textural properties previously described for FC-CSC. Since this is the first time reporting this type of textural evaluation, it was not possible to carry out a comparison with previous work.

\subsubsection{Oil loss determination in sandwich cookies}

An important application of oleogels is their use as an inhibitor of the oil migration in different food formulations. Since the loss of oil from filling cream in cookies can result in an undesirable quality defect, the oil's physical stability in the assembled cookies was determined during 21 days of storage time. Fig. 4 shows that, in general, the amount of oil released increased with time for all samples. After four days of storage, FC-40 showed the highest value of released oil, and significant differences were found between this and the other FC, being FC-40 > FC-30 = FC-26 > FC-CSC. The same trend was observed at subsequent times, with the exception of day 15 at which FC-26 and FC-30 were not significantly different from FC-CSC. At the end of the evaluated 
period, FC-26 and FC-30 showed a reduction of oil loss of 69.7 and $68.3 \%$, respectively, with respect to FC-40, but an increment of 220.3 and $235.3 \%$, respectively, with respect to FC-CSC. Based on these results, it seems like filling creams formulated with oleogel retained the oil to a lesser extent than the control cream. However, it is important to take into account that FC-CSC was not a recently prepared cream since it was used in this evaluation after being in contact with the original cookies. As a consequence, it is not possible to conclude that the profile of released oil found for the commercial cream was "the real" one; even so, it was useful for comparison purposes. Si et al. ${ }^{28}$ investigated the use of oleogels from monoglycerides $(1 \%, 3 \%$, or $6 \%)$ and soybean oil as possible agents of hindering oil migration in commercial confectionery filling for praline products. The authors found that these oleogels had higher migration rates than a commercial confectionery filling fat during the first 10 days of storage, similar rates between 10 and 30 days, and lower rates after storing for 30 days. They explained this different behavior as a direct consequence of insufficient gelation of the oleogels right after preparation of the model praline system followed by a stabilization of the system that allows the crystal network to entrap the liquid oil more efficiently. Tanti et al. ${ }^{17}$ evaluated the oil loss of a shortening control, a commercial sandwich cookie cream, and creams formulated with HPMC and MC oleogels by placing the samples directly on a filter paper. After $24 \mathrm{~h}$, the authors determined oil loss between 3.73 and $7.13 \%$, which are percentages higher than those found in the current work after 21 days. Thus, an important finding to emerge from these comparisons was that some of the formulated MG oleogel-based cookie creams 
were highly stable in terms of oil loss in time, highlighting their potential to be used as filling creams. On the other hand, a correlation between OL and OBC could be expected; however, the same trend with the increase in oleogel content was not found. A possible explanation for these differences may be related to the nature of the tests. OL method quantified the oil released by the migration phenomenon whereas $\mathrm{OBC}$ is a determination based on accelerated oil loss by high-speed centrifugation. Therefore, it seems that the OBC method was more sensitive to detect differences between samples' ability to maintain oil entrapped in their matrix.

\section{Conclusions}

This study demonstrated that oleogels from high oleic sunflower oil and monoglycerides (100 $\mathrm{g} \mathrm{kg}^{-1}$ ) can be used to formulate FC with desirable functionality. The concentration of oleogel played a fundamental role in FC characteristics. FC formulated with $260 \mathrm{~g} \mathrm{~kg}^{-1}$ of oleogel showed viscoelastic moduli values that did not differ significantly from those measured in the commercial cream used as reference. The increase in oleogel content led to the obtention of softer creams but with an increased adhesiveness, producing materials with a better stick capacity that was desirable to achieve the adhesion between cookies. The evaluation of the quality of assembled sandwich cookies indicated that the oil migration from the FC to cookies was low in comparison with data reported in the literature. Moreover, a novel textural evaluation to test assembled cookies revealed that similar work was required to separate cookies containing FC-26, FC-30, and FC-CSC. These positive findings obtained from the full replacement of solid fat for monoglycerides oleogels indicated that it is possible 
to improve the lipid nutritional profile by using oleogels without affecting the quality of the evaluated product. Moreover, this replacement has the added value of using vegetable fat instead of animal fat, which is in line with growing consumer demands for animal-free products. In a further research, sensory evaluation will be addressed to determine consumer acceptability and preferences towards these reformulated healthier products.

\section{Aknowledgment}

The authors acknowledge the financial support by the Consejo Nacional de Investigaciones Científicas y Técnicas (PIP 2015-1122015-0100156-CO), the Agencia Nacional de Promoción Científica y Tecnológica (PICT 2016-0520, PICT 2017-1522), and the Universidad Nacional del Sur (PGI 24/M152) in Argentina.

\section{Conflict of interest}

The authors have no conflict of interest to declare.

\section{References}

1. Bailey RL, Fulgoni VL, Cowan AE, Gaine PC, Sources of added sugars in young children, adolescents, and adults with low and high intakes of added sugars. Nutrients 10:102 (2018).

2. Damen FWM, Luning PA, Fogliano V, Steenbekkers BLPA, What influences mothers' snack choices for their children aged 2-7? Food Qual Prefer 74:10-20 
(2019).

3. Research and Markets,Sweet Biscuit Market - Growth, Trends, And Forecast (2020 - 2025) [Online]. Available from:

https://www.researchandmarkets.com/reports/4771509/sweet-biscuit-marketgrowth-trends-and\#rela3-4602400 [25 August 2020]

4. Stortz TA, Zetzl AK, Barbut S, Cattaruzza A, Marangoni AG, Edible oleogels in food products to help maximize health benefits and improve nutritional profiles. Lipid Technol 24:151-154 (2012).

5. Amrutha Kala AL, Studies on saturated and trans fatty acids composition of few commercial brands of biscuits sold in Indian market. J Food Sci Technol 51:3520-3526 (2014).

6. Doan CD, Tavernier I, Okuro PK, Dewettinck K, Internal and external factors affecting the crystallization, gelation and applicability of wax-based oleogels in food industry. Innov Food Sci Emerg Technol 45:42-52 (2018).

7. World Health Organization, REPLACE Trans Fat-Free by 2023 [Online]. Available from: https://www.who.int/nutrition/topics/replace-transfat [July 10 2019]

8. Patel AR, Alternative Routes to Oil Structuring, 1st edn. Springer Publishing International, New York, New York (2015)

9. Fayaz G, Goli SAH, Kadivar M, Valoppi F, Barba L, Calligaris S, et al., Potential application of pomegranate seed oil oleogels based on monoglycerides, beeswax and propolis wax as partial substitutes of palm oil in functional chocolate spread. 
LWT - Food Sci Technol 86:523-529 (2017).

10. Kaushik I, Organogelation: It’s Food Application. MOJ Food Process Technol 4:66-72 (2017).

11. Giacomozzi AS, Carrín ME, Palla CA, Muffins Elaborated with Optimized Monoglycerides Oleogels: From Solid Fat Replacer Obtention to Product Quality Evaluation. J Food Sci 83:1505-1515 (2018).

12. Singh A, Auzanneau FI, Rogers MA, Advances in edible oleogel technologies A decade in review. Food Res Int 97:307-317(2017).

13. Vernon-Carter EJ, Alvarez-Ramirez J, Meraz M, Bello-Perez LA, Garcia-Diaz S, Canola oil/candelilla wax oleogel improves texture, retards staling and reduces in vitro starch digestibility of maize tortillas. J Sci Food Agric 100:1238-1245 (2020).

14. Franco D, Martins AJ, López-Pedrouso M, Cerqueira MA, Purriños L, Pastrana LM, et al., Evaluation of linseed oil oleogels to partially replace pork backfat in fermented sausages. J Sci Food Agric 100: 218-224 (2020).

15. Adili L, Roufegarinejad L, Tabibiazar M, Hamishehkar H, Alizadeh A, Development and characterization of reinforced ethyl cellulose based oleogel with adipic acid: Its application in cake and beef burger. LWT 126:109277 (2020).

16. Martins AJ, Lorenzo JM, Franco D, Pateiro M, Domínguez R, Munekata PES, et al., Characterization of enriched meat-based pâté manufactured with oleogels as fat substitutes. Gels 6:17 (2020). 
17. Tanti R, Barbut S, Marangoni AG, Hydroxypropyl methylcellulose and methylcellulose structured oil as a replacement for shortening in sandwich cookie creams. Food Hydrocoll 61:329-337 (2016).

18. AOCS, Official methods and recommended practices of the American Oil Chemists’ Society, AOCS Press, 6th edn. Champaign, IL, (2009).

19. ISO/IDF, “Butter, Edible Oil Emulsions and Spreadable Fats-Determination of Fat Content-(Reference Method).” Brussels, (2003).

20. Palla C, de Vicente J, Carrín ME, Gálvez Ruiz MJ, Effects of cooling temperature profiles on the monoglycerides oleogel properties: A rheomicroscopy study. Food Res Int 125:108613 (2019).

21. Bourne MC, Sensory methods of Texture and Viscosity, in Food Texture and Viscosity, 2nd edn., Academic Press, San Diego, pp. 257-291 (2002)

22. Francis FJ, Clydesdale FM, Food colorimetry: theory and applications. AVI Publishing Co. Inc., Westport, Connecticut (1975)

23. Rhim JW, Wu Y, Weller CL, Schnepf M, Physical characteristics of a composite film of soy protein isolate and propyleneglycol alginate. J Food Sci 64:149-152 (1999).

24. Kincs FR, Meat fat formulation. J Am Oil Chem Soc 62:815-818(1985).

25. Patel AR, Babaahmadi M, Lesaffer A, Dewettinck K, Rheological Profiling of Organogels Prepared at Critical Gelling Concentrations of Natural Waxes in a Triacylglycerol Solvent. J Agric Food Chem 63:4862-4869 (2015).

26. Babin H, Dickinson E, Chisholm H, Beckett S, Interactions in dispersions of 
sugar particles in food oils: Influence of emulsifier. Food Hydrocoll 19:513-520 (2005).

27. Co ED, Marangoni AG, Organogels: An alternative edible oil-structuring method. JAOCS, J Am Oil Chem Soc 89:749-780 (2012).

28. Si H, Cheong LZ, Huang J, Wang X, Zhang H, Physical Properties of Soybean Oleogels and Oil Migration Evaluation in Model Praline System. J Am Oil Chem Soc 93:1075-1084 (2016). 


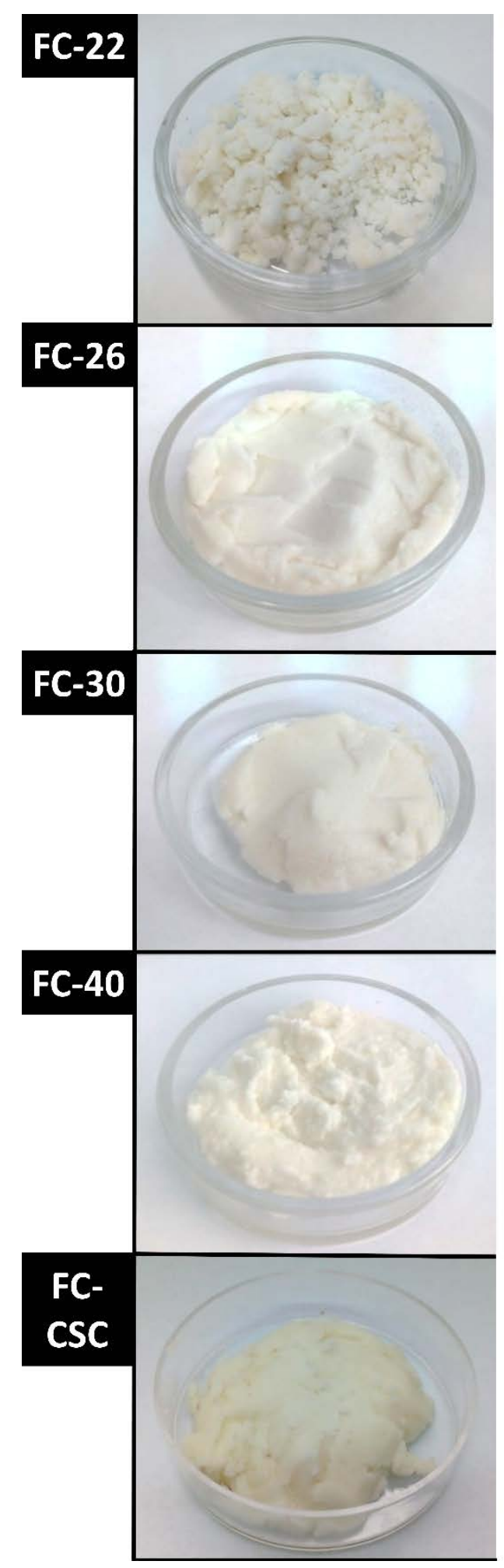

This article is protected by copyright. All rights reserved. 
Figure 1. Images of filling creams formulated with variable amounts of MG oleogel: FC-22 (220 g kg-1), FC-26 (260 g kg-1), FC-30 (300 g kg-1), FC-40 (400 g kg-1), and the commercial filling cream (FC-CSC), which is included for comparison.
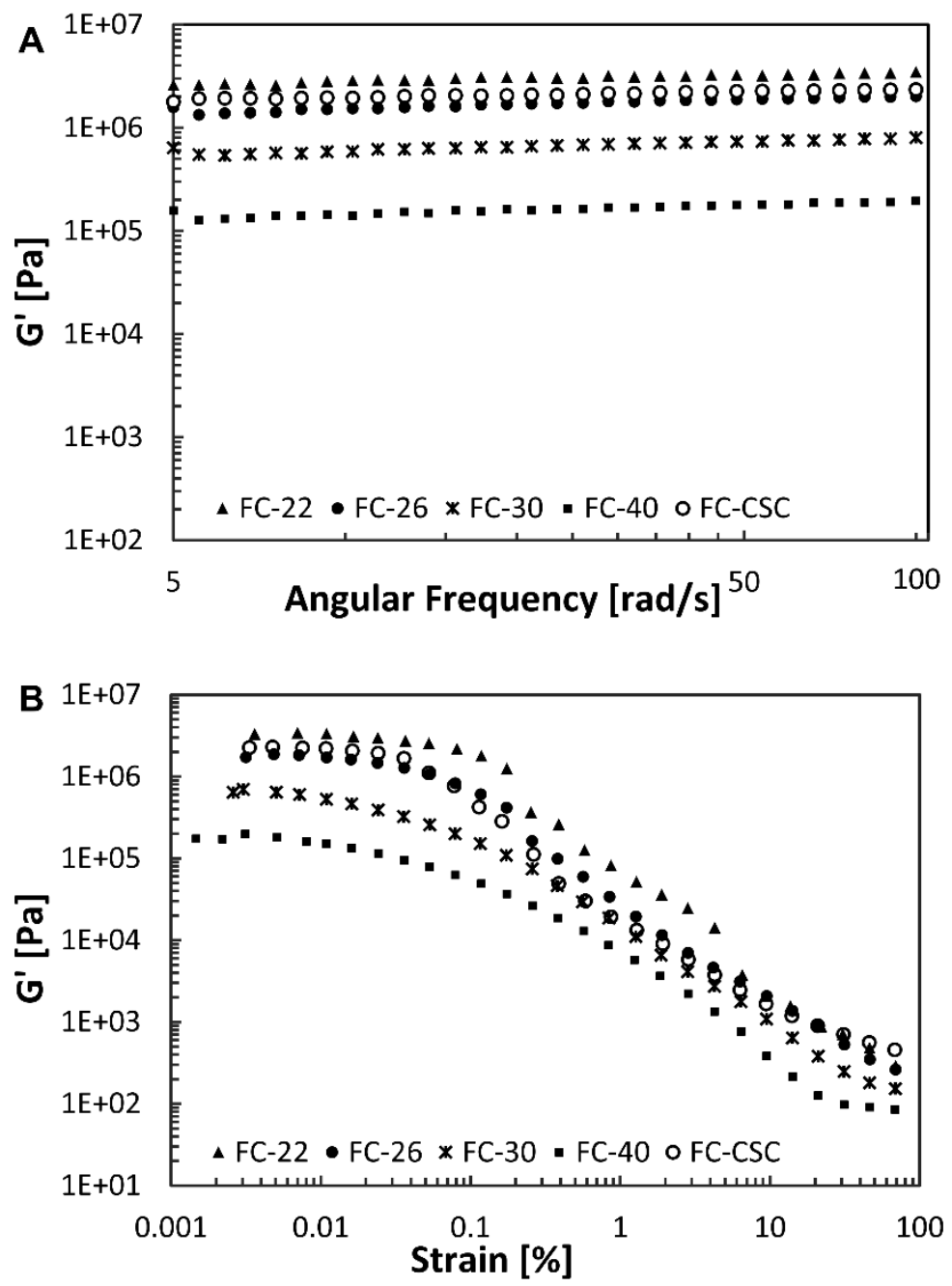

Figure 2. Rheological behavior of filling creams formulated with variable amounts of MG oleogel: A) frequency sweep test and B) strain sweep test. Rheological behavior of FC-CSC is included for comparison. All the curves represent the average behavior over at least three replicates. See nomenclature in Table 1. 
A

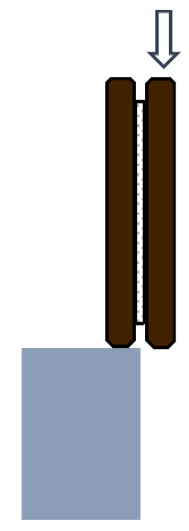

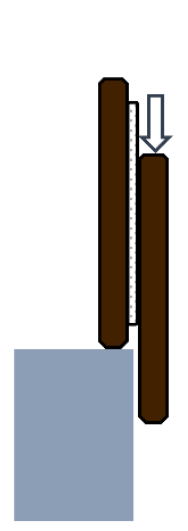

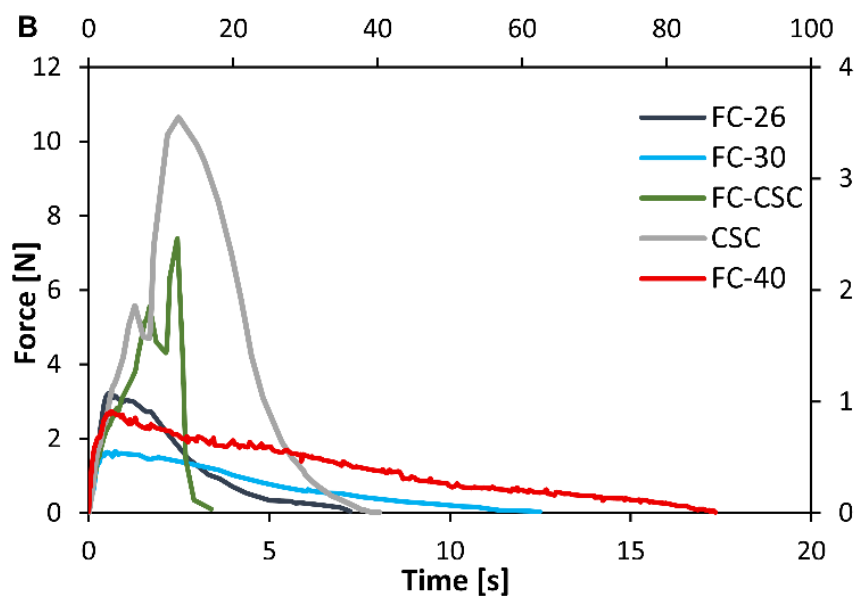

Figure 3. Evaluation of textural properties of assembled sandwich cookies. A) Schematic representation of the performed test in which a tangential force is applied. B) Representative curves obtained from the texture assay. FC-40 is graphed in x- and ysecondary axes for clarity. CSC is included for comparison. See nomenclature in Table 1. 


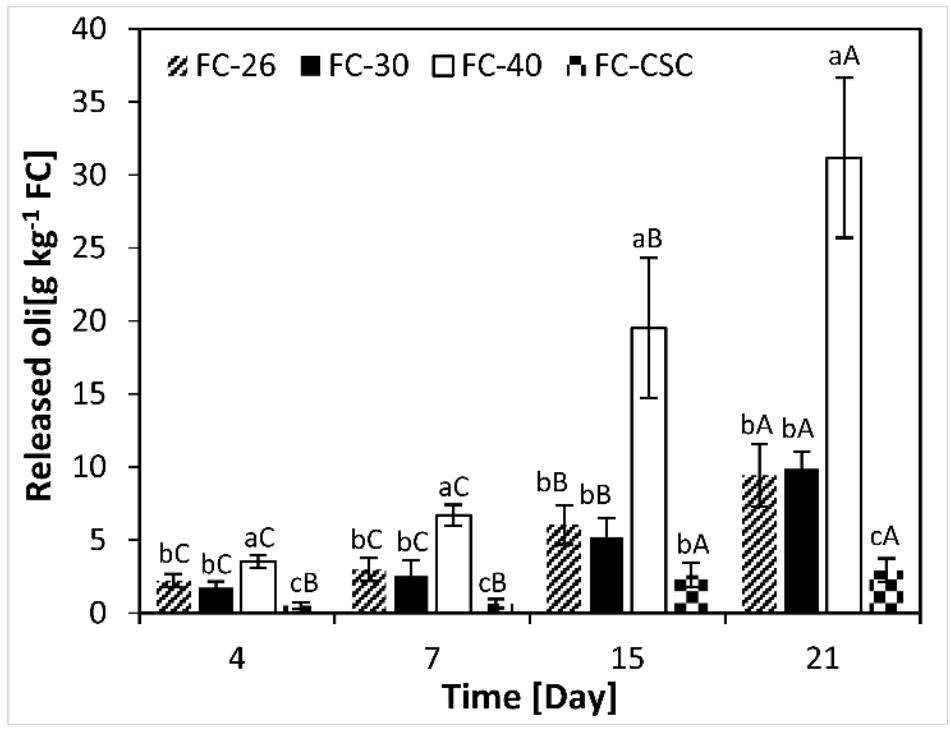

Figure 4. Amount of oil released from assembled sandwich cookies as a function of time. Bars represent means \pm standard deviation. Significant differences between samples are represented by different letters $(\mathrm{p}<0.05)$. The comparison between different FC formulations for a specific time is indicated with lowercase letters and the comparison over time for a specific FC formulation is indicated with uppercase letters. 
Table 1. Formulations and fat content of filling creams elaborated with variable amount of MG oleogel. The commercial cream (FC-CSC) is also included for comparison.

\begin{tabular}{|c|c|c|c|c|c|}
\hline & FC-22 & FC-26 & FC-30 & FC-40 & FC-CSC \\
\hline \multicolumn{6}{|l|}{ Ingredient } \\
\hline Oleogel $\left(\mathrm{g} \mathrm{kg}^{-1}\right)$ & 223.6 & 264.2 & 304.9 & 406.5 & - \\
\hline Sugar $\left(\mathrm{g} \mathrm{kg}^{-1}\right)$ & 725.6 & 685.0 & 644.3 & 542.7 & n.a. \\
\hline Free-fat milk $\left(\mathrm{g} \mathrm{kg}^{-1}\right)$ & 40.7 & 40.7 & 40.7 & 40.7 & n.a. \\
\hline Vanilla essence $\left(\mathrm{g} \mathrm{kg}^{-1}\right)$ & 10.1 & 10.1 & 10.1 & 10.1 & n.a. \\
\hline \multicolumn{6}{|l|}{ Fat content } \\
\hline Total fat $\left(\mathrm{g} \mathrm{kg}^{-1}\right)$ & $223.6 \pm 0.1^{\mathrm{d}}$ & $264.2 \pm 0.1^{\mathrm{c}}$ & $304.9 \pm 0.1^{\mathrm{b}}$ & $406.5 \pm 0.1^{\mathrm{a}}$ & $223.6 \pm 1.3^{\mathrm{d}}$ \\
\hline SFA $\left(\mathrm{g} \mathrm{kg}^{-1}\right)$ & $36.9 \pm 0.3^{\mathrm{e}}$ & $43.6 \pm 0.3^{\mathrm{d}}$ & $48.7 \pm 0.4^{\mathrm{C}}$ & $66.1 \pm 0.5^{b}$ & $111.1 \pm 1.3^{\mathrm{a}}$ \\
\hline MUFA $\left(\mathrm{g} \mathrm{kg}^{-1}\right)$ & $176.6 \pm 0.2^{\mathrm{d}}$ & $208.8 \pm 0.2^{\mathrm{c}}$ & $237.0 \pm 0.3^{b}$ & $322.1 \pm 0.4^{\mathrm{a}}$ & $101.3 \pm 0.9^{\mathrm{e}}$ \\
\hline PUFA $\left(\mathrm{g} \mathrm{kg}^{-1}\right)$ & $10.0 \pm 0.1^{\mathrm{e}}$ & $11.8 \pm 0.1^{\mathrm{C}}$ & $13.6 \pm 0.1^{\mathrm{b}}$ & $18.2 \pm 0.1^{\mathrm{a}}$ & $11.1 \pm 0.3^{\mathrm{d}}$ \\
\hline TFA $\left(\mathrm{g} \mathrm{kg}^{-1}\right)$ & $0.07 \pm 0.00^{\mathrm{e}}$ & $0.08 \pm 0.00^{\mathrm{d}}$ & $0.10 \pm 0.00^{c}$ & $0.13 \pm 0.01^{\mathrm{b}}$ & $10.4 \pm 0.4^{\mathrm{a}}$ \\
\hline
\end{tabular}

SFA: saturated fatty acids; MUFA: monounsaturated fatty acids; PUFA: polyunsaturated fatty acids; TFA: trans fatty acids. n.a. = data not available. Values with different letters within each raw indicate significant differences $(\mathrm{p}<0.05)$. 
Table 2. Tan $\delta$ average value from frequency sweep test and textural properties of formulated and commercial filling creams.

\begin{tabular}{ccccc}
\hline Sample & Tan $\boldsymbol{\delta}$ & Hardness (N) & Adhesiveness (N.s) & Cohesiveness \\
\hline FC-22 & $0.06 \pm 0.01^{\mathrm{d}}$ & $3.48 \pm 0.73^{\mathrm{b}}$ & $0.44 \pm 0.16^{\mathrm{c}}$ & $0.07 \pm 0.02^{\mathrm{c}}$ \\
FC-26 & $0.19 \pm 0.03^{\mathrm{b}}$ & $2.21 \pm 0.23^{\mathrm{c}}$ & $0.55 \pm 0.08^{\mathrm{b}}$ & $0.10 \pm 0.01^{\mathrm{bc}}$ \\
FC-30 & $0.29 \pm 0.01^{\mathrm{a}}$ & $0.66 \pm 0.07^{\mathrm{d}}$ & $0.86 \pm 0.12^{\mathrm{a}}$ & $0.29 \pm 0.04^{\mathrm{a}}$ \\
FC-40 $^{\mathrm{a}}$ & $0.32 \pm 0.03^{\mathrm{a}}$ & n.d. & n.d. & n.d. \\
FC-CSC $^{0}$ & $0.11 \pm 0.02^{\mathrm{c}}$ & $8.67 \pm 1.47^{\mathrm{a}}$ & $0.29 \pm 0.06^{\mathrm{d}}$ & $0.02 \pm 0.00^{\mathrm{cd}}$ \\
\hline
\end{tabular}

n.d. = not determined due to the softness of the material. Different letters represent statistical differences between values across samples. See nomenclature in Table 1. 
Table 3. Physical properties of formulated and commercial filling creams: oil binding capacity (OBC) and color parameters $\left(L^{*}, a^{*}, b^{*}, \Delta E^{*}\right.$, and $\left.W I\right)$.

\begin{tabular}{|c|c|c|c|c|c|c|}
\hline Sample & OBC (\%) & $L^{*}$ & $a^{*}$ & $\boldsymbol{b}^{*}$ & $\Delta \boldsymbol{E}^{*}$ & $W I$ \\
\hline FC-22 & $99.88 \pm 0.09^{a}$ & $81.53 \pm 0.35^{b}$ & $-0.26 \pm 0.04^{b}$ & $10.80 \pm 0.44^{\mathrm{c}}$ & 5.12 & 77.99 \\
\hline FC-26 & $99.85 \pm 0.36^{\mathrm{a}}$ & $81.12 \pm 1.23^{b}$ & $-0.19 \pm 0.10^{\mathrm{b}}$ & $12.21 \pm 0.76^{\mathrm{b}}$ & 3.68 & 77.52 \\
\hline FC-30 & $96.60 \pm 0.52^{b}$ & $81.13 \pm 0.88^{\mathrm{b}}$ & $-0.18 \pm 0.03^{b}$ & $12.70 \pm 0.31^{b}$ & 3.22 & 77.25 \\
\hline FC-40 & $91.78 \pm 0.83^{c}$ & $83.16 \pm 0.29^{a}$ & $-0.30 \pm 0.02^{b}$ & $12.23 \pm 0.12^{\mathrm{b}}$ & 4.49 & 79.18 \\
\hline FC-CSC & $100.06 \pm 0.02^{\mathrm{a}}$ & $80.45 \pm 0.53^{b}$ & $-0.94 \pm 0.18^{a}$ & $15.75 \pm 0.34^{\mathrm{a}}$ & 0.00 & 74.87 \\
\hline
\end{tabular}

Values with different letters within each column indicate significant differences $(\mathrm{p}<0.05)$. See nomenclature in Table 1. 
Table 4. Textural parameters of sandwich cookies assembled with formulated and commercial filling creams.

\begin{tabular}{cccc}
\hline Sample & Adhesion & Fmax (N) & W(N.s) \\
\hline FC-22 & No & n.d. & n.d. \\
FC-26 & Yes & $3.46 \pm 0.35^{\mathrm{c}}$ & $7.68 \pm 3.10^{\mathrm{b}}$ \\
FC-30 & Yes & $1.66 \pm 0.19^{\mathrm{d}}$ & $10.91 \pm 2.73^{\mathrm{b}}$ \\
FC-40 & Yes & $0.85 \pm 0.04^{\mathrm{d}}$ & $34.22 \pm 1.73^{\mathrm{a}}$ \\
FC-CSC & Yes & $8.23 \pm 2.88^{\mathrm{b}}$ & $10.42 \pm 0.99^{\mathrm{b}}$ \\
CSC & Yes & $10.84 \pm 1.73^{\mathrm{a}}$ & $34.99 \pm 3.78^{\mathrm{a}}$ \\
\hline
\end{tabular}

n.d. = not determined. CSC is included for comparison purposes. See nomenclature in Table 1. 